\title{
PAI -1 INHIBITOR AS BIOMARKER OF CARDIORENAL DAMAGE
}

\author{
Danijela D. Tasić $\mathbf{c}^{1,2}$, Katarina S. Tasić $\mathbf{c}^{*}$ \\ ${ }^{1}$ Clinical Center Niš, Clinic of Nephrology, Niš, Serbia \\ ${ }^{2}$ University of Niš, Faculty of Medicine, Niš, Serbia
}

\begin{abstract}
Plasminogen activator inhibitor 1 (PAI-1), which belongs to the family of serine protease inhibitors is the primary regulator of plasminogen activity. PAI-1 is synthesized as a single-chain glycopeptide and is deposited in the platelets in a latent form, from which it is released upon their activation. It is spontaneously converted into stable molecules, unless it reacts with proteins from the plasma. As a powerful inhibitor of fibrinolysis, it participates in the pathogenesis of endothelial damage, processes of accelerated atherosclerosis and thromboembolism. All the diseases of the cardiovascular system which are dominated by the processes of fibrosis and thrombosis lead to an increase in PAI-1 in the circulation. In the emergence and development of atherosclerosis, it plays a role not only in the formation of intraluminal thrombus but also in neointimal proliferation. PAI-1 is not normally present in kidney tissue, but its concentration increases significantly in its acute and chronic kidney disease, thanks to the synthesis by the intrarenally localized inflammatory cells. In addition to genetic predisposition, the factors that directly influence the production of PAI-1 are the following: glycemia, insulin and various neurohumoral factors. It is not normally present in the kidney tissue, but its intrarenal concentration increases significantly in acute and chronic kidney diseases. Numerous studies have confirmed the significant role of PAI-1 in the development of diabetes complications. During the last decade there has been a growth of interest in the introduction of non-invasive methods or biomarkers that would assess the degree of fibrosis in the kidney. Many studies have confirmed association between kidney and heart disease. It is not only that these diseases share common risk factors, but many other mechanisms have been suggested. Plasminogen activator inhibitor -1 plays a role in the pathogenesis of endothelial damage, processes of fibrosis and thrombosis, development of diabetes complications and acute and chronic kidney diseases.
\end{abstract}

Key words: cardio-renal syndrome, biomarkers, plasminogen activator inhibitor-1

\section{Introduction}

Brakman was the first to describe the existence of an inhibitor of tissue plasminogen activator in 1966 and only after 20 years of activator inhibitor-1 tissue plasminogen activator (PAI-1) was isolated from cultures of endothelial cells by which it is named an endothelial type plasminogen inhibitor of tissue. It belongs to the superfamily of serine protease inhibitors - serpin. It has four different structural forms with the conversion of certain regulatory mechanisms of fibrinolytic system. Its structure and site of synthesis (the endothelium, megakaryocytes, endometrium, macrophages, mesothelial cells, adipose tissue) were later determined. The gene responsible for the synthesis of PAI1 was localized to chromosome 7. Once synthesized PAI-1 accumulates in platelets, it can be found in subendothelial matrix and in the bloodstream. Fibrinolytic system includes a wide spectrum of proteolytic enzymes and functions in the process of hemostasis, tissue remodeling, tumor invasion and angiogenesis. The main enzyme in the system is plasminogen activator, plasmin, which is responsible for

Correspondence to: Danijela Tasić, Ph.D.

Clinical Center Niš, Clinic of Nephrology, Niš, Serbia

Phone: +381631094162

E-mail: danijeladt@gmail.com

* Student

Received May $25^{\text {th }}, 2017$, Accepted January $20^{\text {th }}, 2020$ degradation of soluble fibrin degradation products. Activation of plasminogen to plasmin is carried out by two types of activators: urokinase type plasminogen activator (uPA) and tissue-type activator plasminogen (tPA) and fibrin. Clearance and its accumulation are regulated by specific inhibitors of plasminogen activator of which the main is PAI-1. PAI-1 activity-neutralizing factors are XIIa, plasma kallikrein, and XIa [1] (Fig. 1).

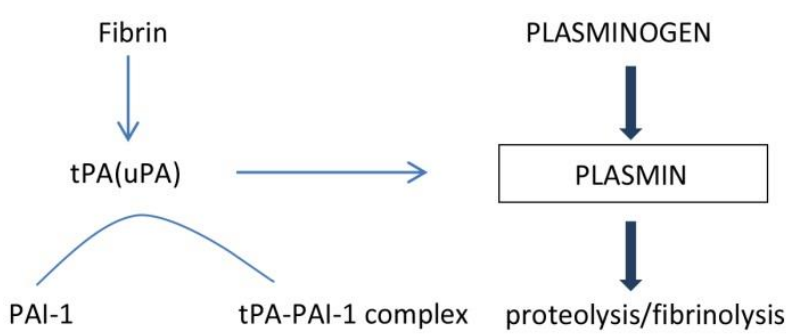

Fig. 1 Schematic presentation of activation of plasminogen

\section{Roles of PAI-1}

In addition to the role of antiprotease, PAI-1 has a high binding affinity for ligands of different receptors. Thus it affects cellular functions such as adhesion, migration, proliferation and intracellular signaling mechanisms that are similar in the cardiac muscle and renal mesangial 
matrix. Changes in the level of PAI-1 are strictly connected to the traditional and non-traditional risk factors and their complications. As an indication of the acute phase of inflammation or the acute inflammatory response, as well as metabolic control and neurohormonal activation, PAI-1 independently or synergistically with other pro-inflammatory molecule is a predictor of cardiovascular risk. In the literature it is described that a high level of PAI-1 is related to atherosclerosis and increased risk for plaque rupture [2]. In contrast to such findings, there are data from some researchers that there is profibrotic effect of PAI-1 in the affected muscles infarction, but also antifibrotic effect in the aging process.

\section{Fibrinolytic System and Heart Disease}

Decreased fibrinolysis with an increased concentration of PAI-1: tPA preformed complex PAI-1 in plasma, occurs in patients with coronary heart disease. The highest expression in the myocardium According to the type of circadian rhythm, the highest expression of PAI1 in the myocardium is in the early morning hours at the exact time when an acute coronary syndrome exhibits a maximum incidence. Locally elevated PAI-1 predisposes the occurrence of the acellular thin atherosclerotic plaque attaching to the wall of the blood vessel and increasing the risk of rupture. When there is a rupture of PAI-1, it is released from the damaged plaque and from the platelet and local concentration of PAI-1 is increased by more than 10 times and a clot is formed. In recent decades, there have been developed percutaneous techniques for the repair of the stenosis of coronary blood vessels (including angioplasty, atherectomy, and stent implantation). Restenosis is associated with different supporting mechanisms involving different hemostatic factors and there is a slow increase in the concentration of PAI-1[3]. On the other hand, TAFI (thrombin activatable fibrinolysis inhibitor) is an antifibrinolytic factor whose growth increases with the influence of procoagulant stimulus but with contradictory research results concerning its association with myocardial infarction. It is clear that there is a direct molecular bond between the TAFI system with coagulation and fibrinolytic cascade [4]. However, the initiation of coagulation under the control of a sound is performed by the endothelial release of TFPI (tissue factor pathway inhibitor) which limits the activity of the TF / VIIa / Xa [5]. The determination of high levels of TAFI and low levels of PAI-1 prior to intervention is important to identify patients at high risk for restenosis. Increased number of late stent thrombosis is explained by the mechanical factor such as a posture in a stent in the penetration of the necrotic part of the tissue, biological factors such as the altered phenotype of endothelial cells and gene polymorphism. Genetic polymorphism explains the relationship between PAI-1 and risk of coronary heart disease. PAI-1 is synthesized and released via the sympathetic-adrenal axis for which it is the key mediator in the stress-induced thrombosis and hypercoagulability conditions [6] (Fig. 2).

\section{Fibrinolytic System and Inflammation}

The complex relationship between inflammation and thrombosis influences the course of coronary artery disease in metabolic syndrome. Proinflammatory particles stimulate multiple prothrombotic effects and acute coronary syndrome. Cardiovascular diseases with altered fibrinolytic system are connected to a raised level of PAI-1. Many authors emphasize the special role of the fibrinolytic system disfunction in metabolic syndrome related to the mechanism of coronary heart disease. The changing role of the fibrinolytic system is in connection with insulin resistance and visceral obesity, where PAI1 plays a crucial role and its concentration rises in the blood and coronary plaque in these patients. The most common disorder of hemostasis, which occurs in these patients is reduced by the level of tissue factor pathway inhibitor, an elevated level of TAFI, VWF, fibrinogen, Factor VII, VIII and XIII and the vitamin K dependent coagulation proteins. Many authors recognize the bidirectional interaction between the elements of the metabolic syndrome and the expression of PAI-1. Adipocyte differentiation in the metabolic syndrome is induced by a set of signaling mechanisms that increase the expression of PAI-1 receptor and interfering with the signaling mechanisms of the insulin, and thus stimulate the formation of obesity [7]. It is assumed that the role of PAI-1 in metabolic syndrome is more important. Therefore, it is attractive as a target for developing new drugs. It has been shown that in the basis of atherosclerosis stands chronic inflammation, and that $\mathrm{C}$-reactive protein (CRP) directly promotes the formation of atherosclerotic lesion through the interaction of the monocyte and endothelial cells by increasing the activity of PAI-1. CRP also increases the activity of PAI-1 in the state of hyperglycemia. On the other hand, CRP reduces the activity of the tissue PA in human endothelial cells which shows that CRP stimulates procoagulant effect (Fig. 3). CRP via activation of the protein kinase and

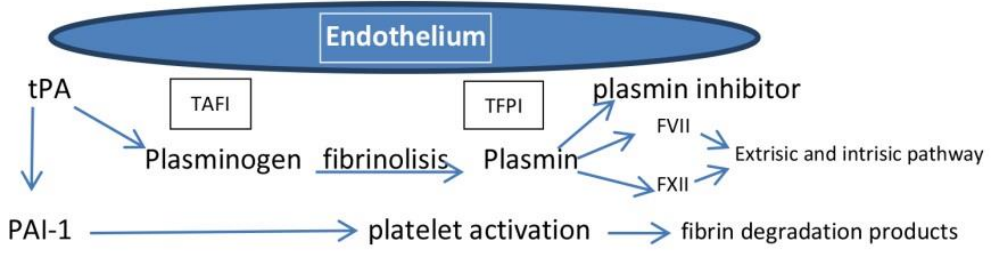

Fig. 2. Schematic presentation of the process of fibrinolysis 


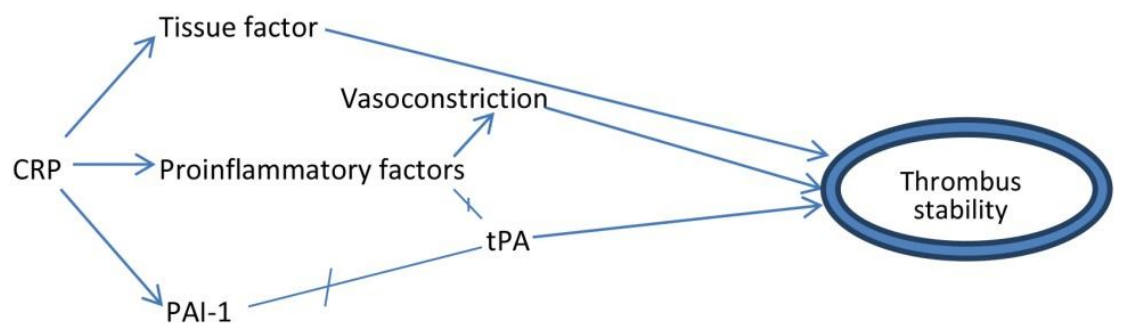

Fig. 3 Schematic presentation of the role of inflammation in the blood vessel reocclusion

NF-kB affects the expression of PAI-1 and affects hemodynamic induced migration of smooth muscle cells in blood vessels [8].

Apart from the acute phase, PAI-1 is increased in conditions of chronic inflammation, which is associated with renal disease. This partly explains the development of accelerated atherosclerosis in this patient population. Kidney diseases in which intra-renal expressions of PAI-1 are increased, are: diabetic nephropathy, focal segmental glomerulosclerosis (FSGS), membranous nephropathy, chronic allograft nephropathy, thrombotic microangiopathy, arteriolonephrosclerosis, "Crescent" focal necrotizing glomerulonephritis, glomerulonephritis. The level of PAI1 is correlated with the severity of disease and in chronic renal disease, it accumulates in the interstitium [9].

\section{Fybrinolitic System and Kidney Disease}

It is believed that PAI-1 plays an important role in the complex mechanisms of tubulointerstitial renal fibrosis. Polymorphism of uric acid affects the plasma concentrations of PAI-1 and is linked with insulin resistance, level of circulating PAI-1 and activation of RAA system. In addition to genetic predisposition, factors which directly influence the production of PAI-1 are: glucose, insulin and various neurohumoral factors. Numerous studies have demonstrated an important role of PAI-1 in the development of diabetic complications [10]. Thrombin stimulates smooth muscle cells in blood vessels that release large amounts of PAI-1. In addition, it is known that the expression of the PAI-1 gene product is closely related to the differentiation of adipocytes and the phenotypic change of smooth muscle cells blood vessels. It is accepted that the PAI-1 risk factor for cardiovascular events in diseases is related with "bad" habits and lifestyle. Fibrosis is important for the development of chronic kidney disease. Decreased renal functional reserve leads to increased activity of inflammation and endothelial dysfunction. These processes increase the risk of the occurrence of cardiovascular disease and venous thrombosis and are associated with elevated levels of PAI-1. Decrease of glomerular filtration rate $(G F R)$ increases the level of molecules which are markers of hemostasis. In addition, impaired renal function contributes to the formation of prothrombotic condition indirectly through the electrolyte disturbances and acid-base status influencing the change of activity of the enzyme coagulation [11]. It was found that atherosclerosis and risk of cardiovascular disease are associated with inflammation and procoagulant state. Patients with GFR $<60 \mathrm{ml} / \mathrm{min} / 1.73 \mathrm{~m}^{2}$ have $6.5 \%$ higher levels of PAI-1 in comparison to subjects with GFR> $90 \mathrm{ml} / \mathrm{min} / 1.73 \mathrm{~m}^{2}$ which indicates that a disturbance homeostasis plays an important role in chronic kidney disease [12]. The fifth stage of chronic kidney disease has a three times higher risk for cardiovascular events compared with the general population while patients on chronic hemodialysis have up to 100 times higher risk of cardiovascular mortality compared with the general population younger than 45 years. Biomarkers of inflammation and oxidative stress are predictors of cardiovascular events in patients on chronic hemodialysis. During hemodialysis, blood in contact with a dialyser activates kallikrein kinin system and induces an inflammatory response by activating leukocytes and the production of cytokines [13]. Cytokines such as TNF alpha, IL 1 beta and IL 6 stimulate the expression of PAI-1 of a large physiological fibrinolysis inhibitor in vivo $[14,15]$. It is well known that a continuous buildup of fibrin deposition in the renal transplant mechanism leads to chronic graft rejection. The mechanism of accumulation of fibrin, tPA is super expressed only in the acute phase of rejection. Upregulation of tPA is present during the progressive stages of chronic rejection and is synchronized with the induction of PAI- 1 in the graft [16]. This means that the induction of PAI-1 is responsible for the accumulation of fibrin resulting in chronic irreversible damage and chronic kidney disease. Testing of PAI-1 genotype is in correlation with development of fibrosis, atrophy of tubules and interstitial fibrosis. It has been shown that genetic variation of fibrinolytic system affects long-term outcome of kidney transplantation. It has been found among people with previous acute rejection that G4 homozygous for the polymorphism had a significantly higher risk of kidney rejection. The conclusion of a number of studies is that determination of the genotype PAI-1 prior to transplantation could help to identify patients who are at risk for chronic rejection of transplanted kidney [17]. PAI-1 is a molecule, which is regulated not only genetically but also by metabolic and inflammatory factors, and it is known that the level of PAI-1 is correlated with the level of decreased function of the graft. 


\section{Conclusion}

Determining prothrombotic biomarkers may help in the planning of timely and appropriate preventive measures for all patients who are at high risk of adverse cardiovascular events. During the last decade there has

\section{References}

1. Jiang Q, Gingles NA, Olivier MA, Miles LA, Parmer JR. The anti-fibrinolytic SERPIN, plasminogen activator inhibitor 1(PAI1 ), is targeted to and released from catecholamine storage vesicles. Blood 2011; 117:7155-7163.

2. $\mathrm{Xu} \mathrm{Z,} \mathrm{Francis} \mathrm{J,} \mathrm{Ploplos} \mathrm{C,} \mathrm{Ploplis} \mathrm{VA.} \mathrm{Plasminogenactivator}$ inhibitor-1 (PAI-1) is cardioprotective in mice by maintaining microvascular integrity and cardiac architecture. Blood 2010; 115:2038-2204

3. Ridker MP, Rifai N, Clearfield M, Downs JR, Weis ES, Miles S et al. Measurement of C-reactive protein for the targeting of statin therapy in the primary prevention of acute coronary events. $\mathrm{N}$ Engl J Med 2001; 344(26):1959-1965.

4. Milijic P, Willemse J, Djordjevic V, Radojkovic D, Colovic M, Elezovic I, et al. Thrombin Activatable Fibrinolysis Inhibitor (TAFI): A Molecular Link Between Coagulation and Fibrinolysis. Srp Arh Celok Lek 2010;138(1):74-78.

5. Van Hinsbergh WMV. Endothelium-role in regulation of coagulation and inflammation. Semin Thromb Hemost 2012; 34:93-106.

6. Flevaris P, Vaughan D. The role of plasminogen activator inhibitor type-1 in fibrosis. Semin Thromb Hemost 2017; 43(2):169-177.

7. Sharony R, Yu PJ, Park J, Galloway CA, Mignatti P, Pintucci G. Protein targets of inflammatory serine proteases and cardiovascular disease. J Inflammat 2010; 7:2-17.

8. Miles LA, Parmer M, Pamer JR. PAI-1: Cardiac friend or foe? Blood 2010; 115:1862-1863.

9. Pretorius M, Donahue BS, Yu Ch, Greelish PJ, Roden DM, Brown NJ. Plasminogen activator inhibitor-1 as a predictor of postoperative atrial fibrillation after cardiopulmonary bypass. Circulation 2007; 116(Suppl 1):1-7. been an increase of interest in the introduction of noninvasive methods or biomarkers that would assess the degree of fibrosis in the kidney. In addition, experimental tests are underway of oral active PAI-1 inhibitor, which is a new class of anti-inflammatory agents.

10. Hillenbrand A, Knippschild $\mathrm{U}$, Weiss $\mathrm{M}$, Schrezenmeier $\mathrm{H}$ Henne-Bruns D, Huber-Lang M, et al. Sepsis induced changes of adipokines and cytokines - septic patients compared to morbidly obese patients. BMC Surgery 2010; 10:26.

11. Tasic D, Radenkovic S, Kocic G, Ilic Deljanin M, Ignjatovic A. Microinflammation Factors in the Common Diseases of Heart and Kidneys. Disease Markers 2015. Article ID 70589. http//dx.doi. org/10.1155/2015/470589.

12. Tasic D. Clinical modalities of the cardiorenal syndrome and the significance of certain biomarkers in its estimation [dissertation on the Internet]. Niš: Universitz of Niš, Faculty of Medicine; 2015. Available from: https://fedorani.ni.ac.rs/fedora/get/o:1027/bdef:Content/ download [in Serbian]

13. Velickovic D, Djukic D, Tasic D. Nonsteroidal anti-inflammatory drugs and hypertension - does an exeption change the rule? Acta Medica Mediane 2014; 53(3): 25-31. doi:10.5633/amm.2014 0304

14. Devaraj S, Xy ZD, Jialal I. C-reactive protein increases plasminogen activator inhibitor-1 expression and activity in human aortic endothelial cells: implications for the metabolic syndrome and atherothrombosis. Circulation 2003; 107:398-404.

15. Zeisberg M, Neilson G. Mechanisms of tubulointerstitial fibrosis J Am Soc Nephrol 2010; 21:1819-1834.

16. Ichimura A, Matsumoto S, Suzuki S, Dan T, Yamaki S, Sato Y, et al. A small molecule inhibitor to plasminogen activator inhibitor 1 inhibits macrophage migration. Arterioscler Thromb Vasc Biol 2013; 33:935-942.

17. Fogo AB. Renal fibrosis: not just PAI-1 in the sky. J Clin Invest 2003; 112:326-328. 